\title{
Cytological diagnosis of infectious conjunctivitis in cats
}

\author{
Sergey Kartashov ${ }^{1, *}$, Anastasia Rakitjanskaya $^{1}$, Marina Petrova $^{1}$, Maria Sultanova ${ }^{1}$, and \\ Maria Oboeva ${ }^{1}$ \\ ${ }^{1}$ Don State Technical University, 344000, Rostov-on-don city, Russian Federation
}

\begin{abstract}
Cytological examination of conjunctival smears obtained from cats with conjunctivitis can reveal the severity and type of inflammation and diagnose conjunctivitis caused by Chlamydophila felis. The diagnosis of chlamydia was made by the study of material taken from the conjunctival sac with a sterile cotton swab by PCR. Material for cytological examination was taken from these cats. Cytological examination of inclusions interpreted as chlamydial, was detected in all cytologic smears from sick cats, and has not been detected in none of healthy cats. Thus, cytological examination is a convenient, non-invasive, easily feasible method, which is suitable for rapid diagnosis of chlamydial conjunctivitis in cats.
\end{abstract}

\section{Introduction}

The conjunctiva is a thin, translucent mucous membrane covering the eyelids (palpebral conjunctiva), the eyeball (bulbar conjunctiva), and the third eyelid (third-century conjunctiva). It is variably pigmented and usually looks smooth and moist. Bright, red blood vessels are visible in non-pigmented areas, indicating its extensive blood supply. The conjunctiva plays an important role in preventing the drying of the cornea and in increasing the mobility of the eyelids and the eyeball. In addition, it is a simple, accessible tissue for sampling and research, as well as a convenient place for drug administration $[1,2,3]$. Interestingly, despite the fact that the conjunctiva is the most vulnerable of all the mucous membranes of the body, it does not remain unprotected. The only lymphatic drainage of the eye is in the conjunctiva. In addition, under the upper and lower eyelids is the conjunctival sac, which produces mucin $[4,5]$. The mucin provides a physical and physiological barrier against microorganisms and foreign bodies by absorbing and consuming as small foreign bodies, and bacteria, as well as providing a medium for the adhesion of the immunoglobulins (i.e. immunoglobulin A) and microbially lysozymes. This latter function is essential, given that the conjunctival sacs contain significant microbial flora, including many potential pathogens microorganism. There are several clinical manifestations suggesting that it is necessary to investigate conjunctival smears. These include: color changes (due to hyperemia, anemia, jaundice or melanosis), any surface irregularities, thickening or mass, inadequate or excessive surface moisture, conjunctival edema

\footnotetext{
* Corresponding author: vera.murgul@mail.ru
} 
(chemosis), sub-conjunctival hemorrhage or emphysema and eye pain (blepharospasm, rubbing) [6-9].

In addition, among the primary objectives in obtaining cytological samples, there is a characteristic discharge from the eyes (serous, mucous or purulent), assessment of inflammatory or neoplastic cells and the identification of infectious organisms, involving the superficial tissues. Finally, the collection and evaluation of conjunctiva cells is recommended for severe, progressive or recurrent lesions of the conjunctiva and in cases that are resistant to empirical treatment. Samples for assessment of conjunctiva can be collected using exfoliative or abrasive methods, aspiration or smear prints. These methods should provide sufficient material for evaluation, maintain morphological integrity, and be neither uncomfortable nor painful for the animal. Additional requirements - ease of implementation and minimal injury $[4,5,10]$.

Conjunctivitis is a common problem in domestic cats. The causes of conjunctivitis in cats are often infections, but conjunctivitis can also be caused by anatomical disorders, trauma or hypersensitivity reactions, these pathologies can develop independently or in various combinations. Sampling for PCR and cytology is an important aspect in the diagnosis of the causes of conjunctivitis, including infectious [2,4,7,11].

Conjunctivitis means inflammation of the conjunctiva; diagnosis - conjunctivitis does not help with the treatment plan. It is necessary to diagnose the cause of inflammation of the conjunctiva, only then it is possible to prescribe treatment correctly. Cats with mucous (or mucopurulent) discharge is quite common, the appearance of discharge from the eyes of serous, mucous or mucopurulent nature does not give clues as to the cause. However, it is reported that, there is a limited number of causes of conjunctivitis in cats. Cats with thickened hyperemic conjunctiva may have a viral infection, chlamydia or eosinophilic conjunctivitis. In this case, with conjunctivitis of any etiology, secondary bacterial conjunctivitis is possible. Determining the cause of cytology is an important aspect of the diagnosis of both the underlying disease and secondary lesions. Conjunctival cytology can be easily performed using simple methods of material selection and staining of smears, and does not require complex equipment and reagents [6-9].

The most common pathogens of infectious conjunctivitis in cats are cat herpesvirus (FHV-1), Chlamydophila felis and Mycoplasma felis [2-4]. Accurate diagnosis allows early to start right and effective treatment, prevent the spread of infection in large populations of cats and to prevent chronic infections and severe complications (symblepharon, blindness, dry kerato-conjunctivitis) $[1,2,3]$. Diagnosis of the disease begins with identification of the pattern of the clinical picture, but the etiology of the disease is impossible to identify the basis of clinical signs. FHV-1 infection can cause conjunctivitis in combination with keratitis and/or respiratory disease, while Chlamydophila felis is considered pathogenic causing primary conjunctivitis, manifested by mucopurulent discharge from the eyes [4,5]. It was reported that Mycoplasma felis causes hyperemia of the conjunctiva and conjunctivitis with mucous secretions [6-9]. However, clinical signs often coincide and conjunctivitis of one etiology can be complicated by other infectious pathogens. Many authors studying this problem note that the clinical picture is not sufficient to differentiate between different infections $[4,5,10]$.

Diagnostic methods needed for an accurate diagnosis of infection FHV-1, Chlamydophila felis and Mycoplasma felis include isolation of the virus by the methods of ELISA, immunohistochemical staining, immunofluorescence analysis, serology studies and PCR $[2,4,7,11]$. PCR analysis has the highest sensitivity as well as high specificity and is generally the preferred method of etiological diagnosis $[4,11]$.

However, PCR results are remote up to a few days (3 to 7), as samples are usually sent to a referral lab. However, the fundamental difference in the treatment of viral and bacterial infections determines the importance of identifying the infectious agent during the initial 
reception of the patient for the appointment of adequate therapy. Cytological examination can be performed during the initial reception of the patient in the clinic, which dramatically increases the diagnostic efficiency and makes it possible to prescribe a full therapy, which determined the relevance of our study.

\section{Aim of this study}

To carry out a cytological study of the conjunctival smear in all cats with a positive result in the PCR reaction to Chlamydophila felis, to describe the cytological picture of the obtained smears, and to determine the diagnostic value of the cytological study in the diagnosis of chlamydial conjunctivitis in cats.

\section{Materials and methods}

The 8 cats were selected for the study with chlamydiosis conjunctivitis patients and 3 healthy cats. All animals underwent complete ophthalmic examination, cytological examination of conjunctival smears and PCR examination for chlamydia.

Ophthalmological examination was carried out with the help of binocular ophthalmic magnifier Magnifier QC Lab, while paying attention to the completeness of the closure of the eye slit, the presence of exophthalmus or enophthalmus, the position and presence of lacrimal points, the state of the lacrimal sac area, determined the cranial reflexes $\mathrm{V}$ pairs of CHF (corneal contact), VII pairs of CHF (pat on the temple).

Tonometry was performed by ophthalmic tonometer "TonoVet".

The following tests were performed: Shirmer I test, tear film break test by Norn, Jones 1 test, fluorescein test, with negative fluorescence test conducted lissamine test.

When inspecting the skin of the eyelids drew attention to the condition of the eyelids, the presence of districi, ectopic eyelashes, the condition of the ducts of the meibomian glands and the nature of the secretions of them, upon inspection of the conjunctiva was noticed discharge from the eyes, swelling of the conjunctiva, type of conjunctival hyperemia, the type of hyperemia of the sclera, in case of necessity conducted a test with erythrina, noted the presence of trichiasis of region of the lacrimal caruncle.

Examination of the cornea was carried out using a slit lamp Shin Nippon XL-1, noted the state of the surface of the cornea, its transparency, the presence of defects, thickenings, overlays, determined the thickness of the precorneal tear film.

Examination of the iris was carried out by a slit lamp Shin Nippon XL-1 for the presence of synechiae, the inversion of the iris pigment sheet was noted the state of the iris vascular apparatus.

Examination of the anterior chamber of the eye was performed by a slit lamp Shin Nippon XL-1 to determine the state of the liquid in the anterior chamber of the eye, the depth of the anterior chamber, the presence of foreign objects, pus, blood in the anterior chamber.

Examination of the pupil and pupillary reactions were determined by white, blue and red colors, the rate of reaction of the pupil to mydriatics was noted.

Examination of the lens was carried out with a slit lamp Shin Nippon XL-1.

Examination of the eye fundus (ophthalmoscopy) was carried out by fundescola Smartscope m-5 for the presence of hyperreflection and hyporeflectivity zones, the presence of hemorrhage, retinal detachment, retinal edema, signs of dysplasia, noted the condition of the retinal vessels, its tortuosity, perivascular edema, hemorrhage and proliferative processes; examination of the optic disc was performed by fundescola Smartscope m-5. 
Ultrasound examination was performed on an ultrasonic scanner DC-N6, using a linear sensor with a frequency of $14 \mathrm{MHz}$. The anterior-posterior size of the eyeball, the thickness of the cornea, the integrity of the cornea, inclusions or foreign objects in the cornea, the anteroposterior size of the anterior chamber of the eye, the contents of the anterior chamber of the eye, the state of the iris and the corneoscleral compound, the state of the ciliary body, topography, shape, transparency and anteroposterior size of the lens, damage to the lens capsule were determined.

Conjunctival smears from 8 cats with conjunctivitis and 3 healthy cats were stained according to Romanovsky-Gimza and assessed by cytological method the type of inflammation, inclusions characteristic for Chlamydophila felis and other cytological changes characteristic of this infection.

Cytological assessment. In the presence of a moderate or significant number of inflammatory cells, a differential count of 200 cells was performed. Inflammation was classified as neutrophil ( $>90 \%$ neutrophils), lymphocytic ( $>50 \%$ lymphocytes) or mixed (neutrophils and lymphocytes, neutrophils $\leq 90 \%$ and lymphocytes $\leq 50 \%$ ). Eosinophils, mastocytes and macrophages were not found in our study in cytological smears.

Inclusions in the cytoplasm of epithelial cells were considered chlamydial if they had the following morphology: coccoid basophilic corpuscles $0.5-1 \mu \mathrm{m}$ in diameter elementary corpuscles; intermediate corpuscles, had a morphology of amorphous or finely dispersed single basophilic inclusions in the cytoplasm 1-3 $\mu \mathrm{m}$ in diameter [18]. Reticular cells had a morphology of structures containing many small elementary bodies, usually covering the cell nucleus.

\section{Results and discussion}

All cats infected with confirmed chlamydia were noted epiphora, intermittent blepharospasm, swelling of the conjunctiva, until the development of hamasa and secretion from grey to green (figures 1,2). Some cats had positive test fluorescently, allowing these cats to diagnose kerato-conjunctivitis sicca. Lesions of the respiratory tract in these cats were absent. Thus, all cats with chlamydia were diagnosed as follows: catarrhal and catarrhal purulent conjunctivitis or kerato-conjunctivitis. 8 cats clinically ill with chlamydia were selected for the study, the diagnosis was confirmed by PCR. Clinically healthy cats were negative in PCR not only for chlamydia, but also for the entire respiratory profile (FHV-1, Chlamydophila felis, Mycoplasma felis) (table 1).

Table 1. Age and detection of Chlamydophila felis by PCR analysis in healthy control cats and in cats with conjunctivitis.

\begin{tabular}{|c|c|c|}
\hline \multirow{2}{*}{} & \multicolumn{2}{|c|}{ Cats } \\
\cline { 2 - 3 } & $\begin{array}{c}\text { Control, healthy, negative by } \\
\text { PCR }\end{array}$ & Chlamydophila felis positive cats \\
\hline $\begin{array}{c}\text { Number of } \\
\text { cats }\end{array}$ & 3 & 8 \\
\hline Age & $0.2-1.5$ & $0.2-1.5$ \\
\hline
\end{tabular}

In the cytological picture of healthy cats, layers and palpebral structures of welldesigned prismatic cells of palpebral epithelium prevailed, signs of inflammation were not noted, neutrophils and other cells except epithelial were absent. The cytoplasm of palpebral epithelium cells was uniformly colored, weakly basophilic without any inclusions (figure 3, 4).

Table 2. Cytological data in cats with conjunctivitis.

\begin{tabular}{|l|l|l|}
\hline & Healthy cats, control & Cats with conjunctivitis \\
\hline
\end{tabular}




\begin{tabular}{|c|c|c|}
\hline & & Chlamydophila felis positive \\
\hline Number of cats & 3 & 8 \\
\hline $\begin{array}{c}\text { no inflammation, or marked } \\
\text { inflammation }\end{array}$ & $3 / 100 \%$ & $0 / 0 \%$ \\
\hline $\begin{array}{c}\text { severe inflammation, } \\
\text { (inflammation cells up to 10 } \\
\text { in each field of view) }\end{array}$ & - & $8 / 100 \%$ \\
\hline neutrophil inflammation & - & $6 / 75 \%$ \\
\hline lymphocytic inflammation & - & $2 / 25 \%$ \\
\hline mixed inflammation & - & $2 / 25 \%$ \\
\hline presence of eosinophils & - & $0 / 0 \%$ \\
\hline dual core cell & - & $2 / 25 \%$ \\
\hline epithelial dysplasia & - & $2 / 25 \%$ \\
\hline bacterial conjunctivitis & - & $0 / 0 \%$ \\
\hline
\end{tabular}

Flat cells of the cornea showed the cytoplasm from slightly basophilic to suboccipital, inclusions, and signs of degeneration or dysplasia of the epithelium was absent (table 2).

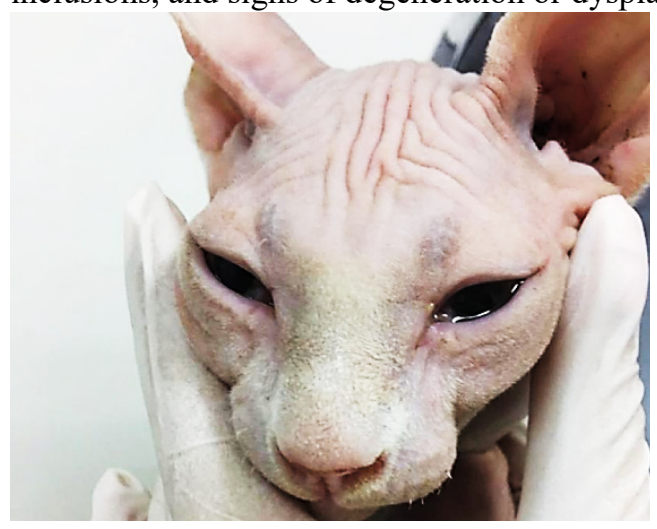

Fig. 1. Epiphora and blepharospasm in a cat with chlamydia.

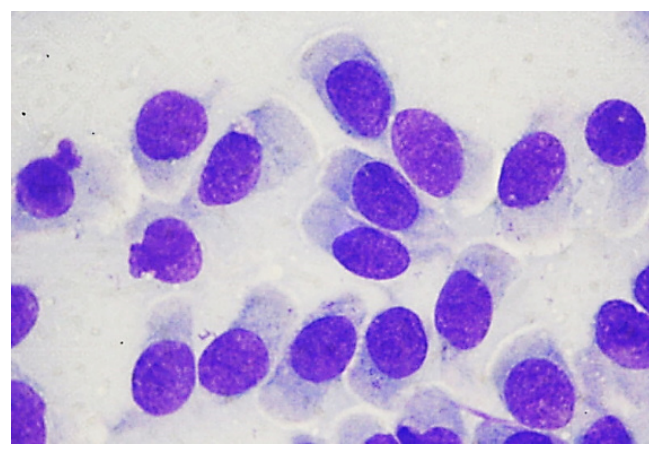

Fig. 3. Palpebral epithelium of a healthy cat.

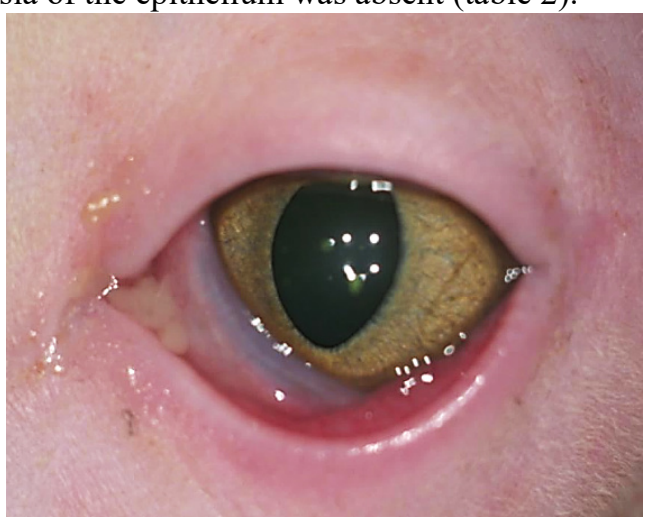

Fig. 2. Redness, swelling of the conjunctiva and purulent discharge from cats sick with chlamydia.

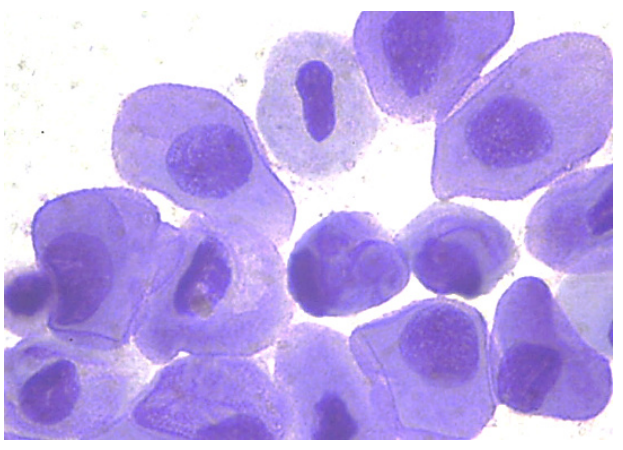

Fig. 4. Flat epithelium of the cornea of a healthy cat. 


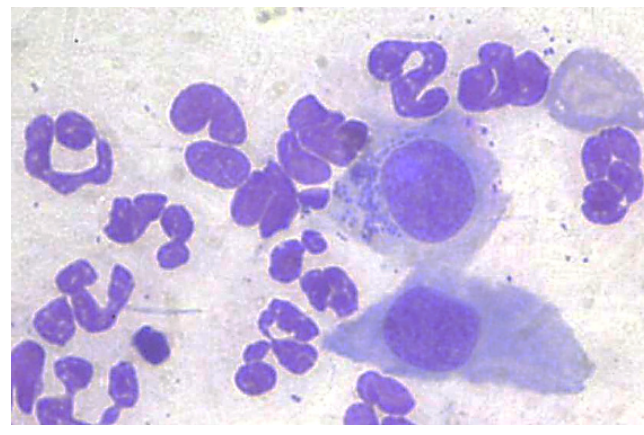

Fig. 5. Expressed neutrophilic inflammation and the accumulation of the elementary Taurus Chlamydophila felis.

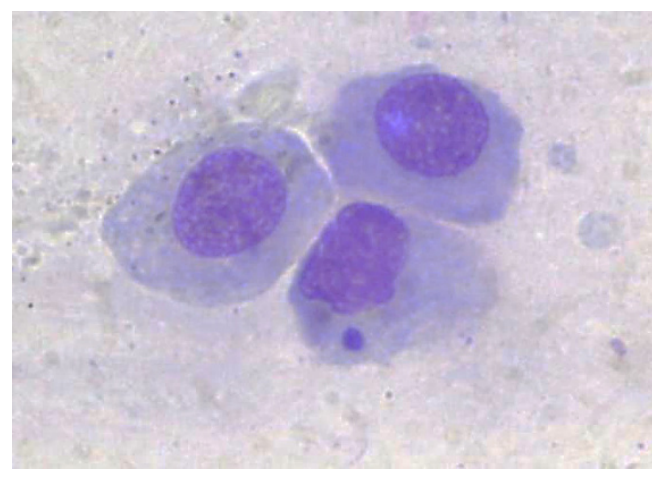

Fig. 7. The intermediate body of Chlamydophila felis in a flat cell of the cornea.

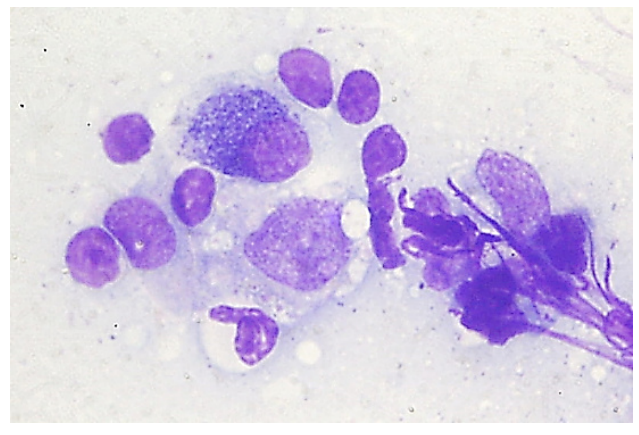

Fig. 6. Reticular body, near the nucleus of palpebral cells, two monocytes with phagocytized elementary bodies.

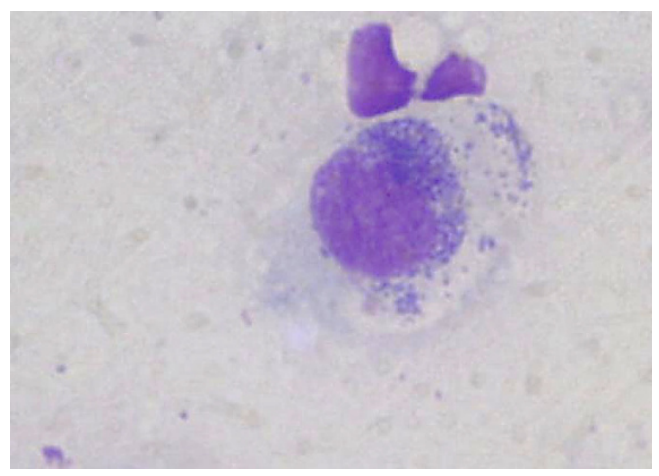

Fig. 8. Reticular bodies near the nucleus and elementary bodies on the periphery of the epithelial cell.

All cats infected with Chlamydophila felis had many inflammatory cells, mainly neutrophils, in the cytological smear (figure 5), confirming the conclusion that the absence of inflammation makes the diagnosis of chlamydial conjunctivitis unlikely [11]. It has been reported that Chlamydophila felis causes neutrophilic inflammation in the early stages of the disease, with mononuclear cells (macrophages) that appear as the process of chronicles (figure 6) [18]. In some cats, the smear was dominated by lymphocytes, and this inflammation was interpreted as lymphocytic (table 2). However, this type of inflammation should be considered with caution, because the lymphocytes found in the sample from the conjunctiva may represent normal follicular tissue, and not lymphocytic inflammation. Correct collection of the material, without affecting the inner surface of the third eyelid will help to avoid such errors. In general, all cats with chlamydia noted neutrophilic or mixed neutrophilic-lymphocytic inflammation (table 2).

Chlamydial cytoplasmic inclusions found in the conjunctival smear can be interpreted as three different stages of intracellular development of the pathogen.

Chlamydial cytoplasmic inclusions of dark blue color rounded, fine-grained structures from 5 to $10 \mu \mathrm{m}$ and more located near the nucleus, in some cases covering it, are interpreted as reticular bodies of Chlamydophila felis (figures 6, 8).

Along with reticular bodies in individual cells, dark blue inclusions from 1 to $2 \mu \mathrm{m}$ in diameter are observed separately located throughout the cytoplasm, and are interpreted as elementary bodies of Chlamydophila felis (figures 5,8). Basophilic amorphous inclusions up to 2-3 microns in diameter were observed only in some cells, usually single, interpreted 
as intermediate bodies (figure 7). In this study, reticular and elementary bodies prevailed. Elementary and intermediate bodies in the absence of reticular bodies should be interpreted with caution, since they can be associated with inclusions of another nature: mucin, Mycoplasma felis, degradable nuclei or tissue detritus.

The inclusions of Chlamydophila felis are easiest to detect at an early stage of the disease and as it have been reported inclusions disappear within 14 days after inoculation, even when clinical signs persisted for several more weeks [18]. All cats with chlamydia were studied within 14 days from the start of the disease, which is consistent with the literature studied by us, the inclusions were found in the acute phase of infection [10]. We do not agree with the authors of another study, which indicates that the cytological study had limited use for the diagnosis of chlamydia in cats, apparently this restriction is associated with chronic chlamydial conjunctivitis [2]. There are reports that in order to avoid diagnostic errors, it is necessary in the interpretation of chlamydial inclusions clearly adhere to the morphological criteria described, in addition, if similar inclusions are present only in a few cells of the test material, the diagnosis is critical, if more than $10 \%$ of epithelial cells in the drug have characteristic cytoplasmic inclusions, the diagnosis becomes more obvious, special attention should be paid to the presence of reticular bodies.

\section{Conclusion}

Cytological examination of conjunctival smear in chlamydia conjunctivitis in cats is a valuable method of diagnosis of the disease. All signs of inflammation are completely absent in healthy cats, and in the preparation, there are only epithelial cells of palpebral and corneal epithelium having a stereotypical morphology, which eliminates the disease. In the presence of a pronounced inflammatory process, usually neutrophil, and cytoplasmic inclusions characteristic for reticular cells in more than $10 \%$ of epithelial cells, the diagnosis of chlamydia is obvious.

The inclusions of Chlamydophila felis are easiest to detect at an early stage of the disease and, they disappear within 14 days after infection of the animal, even when clinical signs persisted for a few more weeks. In cats with chlamydia, during the first 14 days from the start of the disease, cytological examination may be the main method of diagnosis, whereas in case of chronic chlamydia, it is possible to use other methods of diagnosis.

The gold standard for the diagnosis of chlamydia is PCR, but the duration of this study is not always convenient in the clinical practice of a veterinarian. With an obvious cytological diagnosis, it is possible to early prescribe the correct treatment, which avoids unnecessary polypragmasia, which is especially important for young patients. With a difficult cytological diagnosis, PCR is considered as a diagnostic method of choice. Thus, cytological examination is a convenient, non-invasive, easily feasible method, which is suitable for rapid diagnosis of chlamydial conjunctivitis in cats.

\section{References}

1. B. Strom Holst, J. Frossling, J. Feline Med Surg., 11, pp. 793-802 (2009)

2. A. Hartmann, J. Hawley, C. Werckenthin, M. Lappin, K. Hartmann, J. Feline Med Surg., 12, pp. 775-782 (2010)

3. P. Steagall, B. Monteiro-Steagall, P. Taylor, J. Vet Intern Med., 28, pp. 762-770 (2014)

4. R. Gaskell, S. Dawson, A. Radford, Infectious diseases of the dog and cat. St Louis: Elsevier Saunders, 4 ed., pp. 151-162 (2012) 
5. A. Hartmann, J. Hawley, C. Werckenthin, M. Lappin, K. Hartmann, J. Feline Med Surg., 12, pp. 775-782 (2010)

6. K. Masubuchi, A. Wakatsuki, K. Iwamoto, T. Takahashi, T. Kokubu, M. Shimizu, J. Feline Med Surg., 12, pp. 609-613 (2010)

7. R. Burns, D. Wagner, C. Leutenegger, P. Pesavento, J. Clin Microbiol, 49, pp. 2454- 2460 (2011)

8. T. Gruffydd-Jones, D. Addie, S. Belak, C. Boucraut-Baralon, H. Egberink, T. Frymus, J. Feline Med Surg., 11, pp. 605-9 (2009) doi:10.1016/j. jfms.2009.05.009

9. R. Raskin, D. Meyer, Canine and Feline Cytology. A Color Atlas and Interpretation Guide. St Louis, MO: Saunders Elsevier, 2nd ed., pp. 367-369 (2010)

10. H. Strom, L. Krook, S. Englund, A. Lagerstedt, G. Bolske, J. Vet Res., 72, pp. 806$812(2011)$

11. R. Soderlund, G. Bolske, R. Holst, A. Aspan, J. Vet Diagn Invest., 23, pp. 890-893 (2011) 